\title{
Factors Influencing the Electrospray Intrasource Separation and Selective Ionization of Glycerophospholipids
}

\author{
Xianlin Han,* Kui Yang, Jingyue Yang, Kora N. Fikes, Hua Cheng, \\ and Richard W. Gross, ${ }^{\dagger \ddagger}$ \\ Division of Bioorganic Chemistry and Molecular Pharmacology, Washington University School of Medicine, \\ St. Louis, Missouri, USA
}

\begin{abstract}
The external electric field induces a separation of cations from negative electrolyte ions in the infusate while differential ionization of molecular species that possess differential electrical propensities can be induced in either the positive- or negative-ion mode during the electrospray ionization process. These physical and electrical processes that occur in the electrospray ion source have been used to selectively ionize lipid classes possessing different electrical propensities that are now known as "intrasource separation and selective ionization". However, the chemical principles underlying charge-dependent alterations in ionization efficiencies responsible for the selective ionization of lipid classes are not known with certainty. Herein, we examined the multiple factors that contribute to intrasource separation and selective ionization of lipid classes under optimal instrumental conditions. We demonstrated that many different lipid classes could be selectively ionized in the ion source and that intrasource resolution of distinct molecular constituents was independent of lipid concentration, flow rate, and residual ions under most experimental conditions. Moreover, the presence of alkaline conditions facilitates the selective ionization of many lipid classes through a mechanism independent of the design of the ESI ion source. Collectively, this study provides an empirical foundation for understanding the chemical mechanisms underlying intrasource separation and selective ionization of lipid classes that can potentially be used for global analysis of cellular lipidomes without the need for chromatographic separation. (J Am Soc Mass Spectrom 2006, 17, 264-274) (C 2006 American Society for Mass Spectrometry
\end{abstract}

S ince Fenn and colleagues [1, 2] initially developed the electrospray ionization (ESI) technique for the mass spectrometric analysis of polar compounds, many theories of the physical processes involved in ion generation during ESI have been proposed and validated in some detail [3-8]. The two main stages in the mechanism of ion generation are the production of the charged droplets from the sprayed solution in the presence of high electric fields and the formation of gas-phase ions from the dispersed charged droplets [9]. Although many physicochemical features of the ionization process are still being actively investigated, clearly the physical properties of an analyte and the effects resulting from surface tension and surface charge interactions in the sprayed droplets play important roles in the two main stages of the ionization process.

Published online January 18, 2006

Address reprint requests to Dr. X. Han, Washington University School of Medicine, Campus Box 8020, 660 South Euclid Ave., St. Louis, MO 63110, USA.E-mail: xianlin@wustl.edu

* Also at the Department of Medicine, Washington University School of Medicine, St. Louis, MO

${ }^{+}$Also at the Department of Molecular Biology and Pharmacology, Washington University School of Medicine, St. Louis, MO

$\ddagger$ Also at the Department of Chemistry, Washington University, St. Louis, $\mathrm{MO}$
An essential feature of electrospray ionization is that charge separation occurs in molecules containing an ionic bond in the ion source under high electrical potentials (typically $\sim 4 \mathrm{kV}$ ) $[9,10]$. Specifically, if there are both positively and negatively charged moieties present in the infused solution, then in the positive-ion mode, the electrospray ion source selectively generates cations in the gas phase whereas the anions are selectively left at the end of the spray capillary or dispersed as electrically neutral molecules in the gas phase and are eventually disposed of as waste after oxidation/reduction reactions [11]. Similarly, in the negative-ion mode, the ion source selectively generates anions in the gas phase and removes the cationic moieties to waste after redox chemistry has occurred. Therefore, an electrospray ion source is functionally analogous to an electrophoretic separation conducted with a continuously equilibrating mobile phase [9-11]. In fact, an electrospray ion source is more broadly applicable than an electrophoretic cell to resolve multiple types of analytes since the ion source can generate gas-phase ions from electrically neutral but polar compounds. Thus, when the analytes in the infused solution do not carry separable charge(s) but possess intrinsic dipoles, these compounds can be induced to interact with small cation(s) or anion(s) available in the matrix to yield adduct ions in the positive- 
or negative-ion mode, respectively, in a high electric field. The ionization efficiencies of these electrically neutral compounds depend on their inherent dipoles, the concentration of the small matrix ions, the affinity of the small ions for the analytes, and the electrochemical properties of the resultant adducts [12].

We recognized the potential application of these physical processes involved in electrospray ionization of lipids in our earliest study on lipid analysis by ESI/MS [13], and applied these principles to determine phospholipid classes and individual molecular species directly from chloroform extracts [14]. Based on the physical processes inherent in ESI, different lipid classes possessing disparate electrical properties can be functionally separated and selectively ionized in the ion source. The principles underlying these processes are entirely analogous to the ion-exchange chromatographic techniques previously employed for separation of naturally occurring phospholipid classes [15]. Thus, this process has been termed "intrasource separation and selective ionization" since it takes place in the ion source of the mass spectrometer [12, 16-18]. Although it has been functionally characterized in some detail [12, 18], the factors that mediate these physical processes have not been identified. Accordingly, because of their influence on the selective ionization of lipid classes in the ion source, an improved understanding of these factors is critical to advance the analyses of complex lipid mixtures and to facilitate the high throughput and automated analysis of lipids.

Herein, we used systematic mixtures of synthetic glycerophospholipids to examine the factors underlying the intrasource separation and selective ionization of different lipid classes and identified the critical physical and/or chemical interactions which influence the degree of these processes. We demonstrated that intrasource separation and selective ionization of phospholipid classes was independent of the lipid concentration and the infusion flow rate within a wide range of experimental conditions, but the observed selective ionization was dependent on the number of different individual molecular species that comprise each phospholipid class with specific charge characteristics in the infusion solution. Thus, through examination of multiple physical and chemical factors influencing the intrasource separation and selective ionization of phospholipid classes, this study provides an empirical foundation for understanding the chemical mechanisms underlying intrasource separation and selective ionization of lipid classes that can be potentially used for global analysis of cellular lipidomes without the need for chromatographic separation.

\section{Materials and Methods}

\section{Materials}

Synthetic phospholipids including choline glycerophospholipids (PCs) (1,2-dimyristoleoyl-sn-glycero3-phosphocholine and 1,2-dioleoyl-sn-glycero-3- phosphocholine (14:1-14:1 and 18:1-18:1 PtdCho, respectively), ethanolamine glycerophospholipids (PEs) (1,2-dimyristoyl-sn-glycero-3-phosphoethanolamine, 1,2- dipentadecanoyl-sn-glycero-3-phosphoethanolamine, 1,2-dioleoyl-sn-glycero-3-phosphoethanolamine, and 1,2-diarachidonoyl-sn-glycero-3-phosphoethanolamine (14:0-14:0, 15:0-15:0, 18:1-18:1, and 20:4-20:4 PtdEtn, respectively), and phosphatidylglycerols (PtdGro) (1,2-dimyristoyl$s n$-glycero-3-phosphoglycerol, 1,2-dipentadecanoyl-sn-glycero-3-phosphoglycerol, 1,2-dioleoyl-sn-glycero-3-phosphoglycerol, and 1,2-didecosanoyl-sn-glycero-3-phosphoglycerol (14:0-14:0, 15:0-15:0, 18:1-18:1, and 22:6-22:6 PtdGro, respectively) were purchased from Avanti Polar Lipids, Inc. (Alabaster, AL). All phospholipids were quantitated by capillary gas chromatography after methanolysis [19]. Solvents for sample preparation and for mass spectrometric analysis were obtained from Burdick and Jackson (Honeywell International Inc., Burdick and Jackson, Muskegon, MI). All other chemical reagents were at least analytical grade or the best grade available and obtained from either Fisher Scientific (Pittsburgh, PA) or Sigma-Aldrich Chemical Company (St. Louis, MO).

\section{Sample Preparation}

A stock solution of each phospholipid sample in 1:1 of chloroform/methanol (vol/vol) was prepared and stored under nitrogen at $-20^{\circ} \mathrm{C}$. The solutions were brought to room temperature just before use. The mixtures of phospholipids at various concentrations/ compositions were prepared from these stock solutions using gas-tight syringes. All the mixed solutions were extracted twice by a modified Bligh and Dyer technique [20], utilizing either $50 \mathrm{mM} \mathrm{LiCl}$, tenfold diluted phosphate buffered saline (PBS), $50 \mathrm{mM}$ ammonium acetate, or $50 \mathrm{mM} \mathrm{NH}_{4} \mathrm{Cl}$ in an aqueous layer. The extracts were dried under a nitrogen stream, dissolved in chloroform, filtered with $0.2 \mu \mathrm{m}$ Gelman acrodisc CR PTFE syringe filters (Gelman Science, Ann Arbor, MI), and dried under a nitrogen stream. The dried phospholipid mixtures were resuspended in $0.5 \mathrm{~mL}$ of $1: 1$ ( $\mathrm{vol} / \mathrm{vol}$ ) chloroform/methanol, stored at $-20^{\circ} \mathrm{C}$ after gently flushing with nitrogen, and used within a week after preparation.

\section{Electrospray Ionization Mass Spectrometry of Lipids}

Most of ESI/MS analyses were performed utilizing a triple-quadrupole mass spectrometer (ThermoElectron TSQ Quantum Ultra, San Jose, CA) equipped with an electrospray ion source as described previously [21, 22]. All ESI/MS analyses of lipids were conducted by direct infusion employing a Harvard syringe pump at the indicated flow rates. The total lipid concentration of the infused solution was varied from 0.05 to $104 \mathrm{pmol} / \mu \mathrm{L}$ as indicated. Typically, a 1-min period of signal aver- 
aging in the profile mode was employed for each MS spectrum and a 2-min period of signal averaging for each product ion MS spectrum was employed. Product ion ESI/MS analyses of phospholipids were similarly performed as described previously [23].

ESI/MS analyses were also performed utilizing a QqTOF mass spectrometer (Applied Biosystems/MDS Sciex QStar XL, Concord, Canada) equipped with an ionspray ion source. The instrument was operated under Analyst QS software. The following instrumental settings were employed: spray voltage, $\pm 4500 \mathrm{~V}$; declustering potential, $\pm 20 \mathrm{~V}$; focusing potential, $\pm 150 \mathrm{~V}$; declustering potential $2, \pm 5 \mathrm{~V}$; focusing rod offset, \pm 15 ; ion source gas, scale 2; curtain gas, scale 20; collision gas, 2 mTorr; ion release delay, $2 \mathrm{mS}$. All ESI/MS analyses of lipids were conducted by direct infusion employing a Harvard syringe pump at the indicated flow rates. The total lipid concentration of the infused solution was varied from 0.01 to $104 \mathrm{pmol} / \mu \mathrm{L}$ as indicated. Typically, a 1-min period of signal averaging in the profile mode was employed for each MS spectrum.

\section{Results and Discussion}

\section{Intrasource Separation and Selective Ionization of Phospholipid Classes}

Model mixtures of synthetic phospholipids comprised of three representative categories of phospholipid classes with distinct electrical propensities were employed. The first category, represented by PtdGro species, was anionic lipids. The second category, represented by PtdCho, was electrically neutral but zwitterionic lipids under alkaline conditions. The third category, represented by PtdEtn, was lipids whose electrical properties were easily modified by the presence of a small amount of alkali. Two individual molecular species at equimolar concentrations from each of these phospholipid classes were selected to represent the variations in chain length and unsaturation typically found in biological phospholipids. Most of the experiments were performed using phospholipid mixtures composed of PtdGro, PtdCho, and PtdEtn at a molar ratio of 1:10:15, which was approximately the molar ratio of these three classes of phospholipid in biological samples [24]. In addition, lipid analysis after intrasource separation and selective ionization was conducted in the low concentration region since many studies have demonstrated that the linear dynamic range for quantitative analysis of lipids occurs in the region of $<50 \mathrm{pmol}$ of a lipid class/ $\mu \mathrm{L}$ in chloroform/ methanol (1:1, vol/vol) [13, 25-27].

Negative-ion ESI/MS analyses of mixtures at different concentrations were performed by direct infusion into the ion source at a flow rate of $8 \mu \mathrm{L} / \mathrm{min}$. Mass spectra demonstrated two very abundant and four low-abundance ion peaks with the base peak of $\sim 7.8 \times$ $10^{6}$ ion current (Figure 1a). Tandem MS analyses
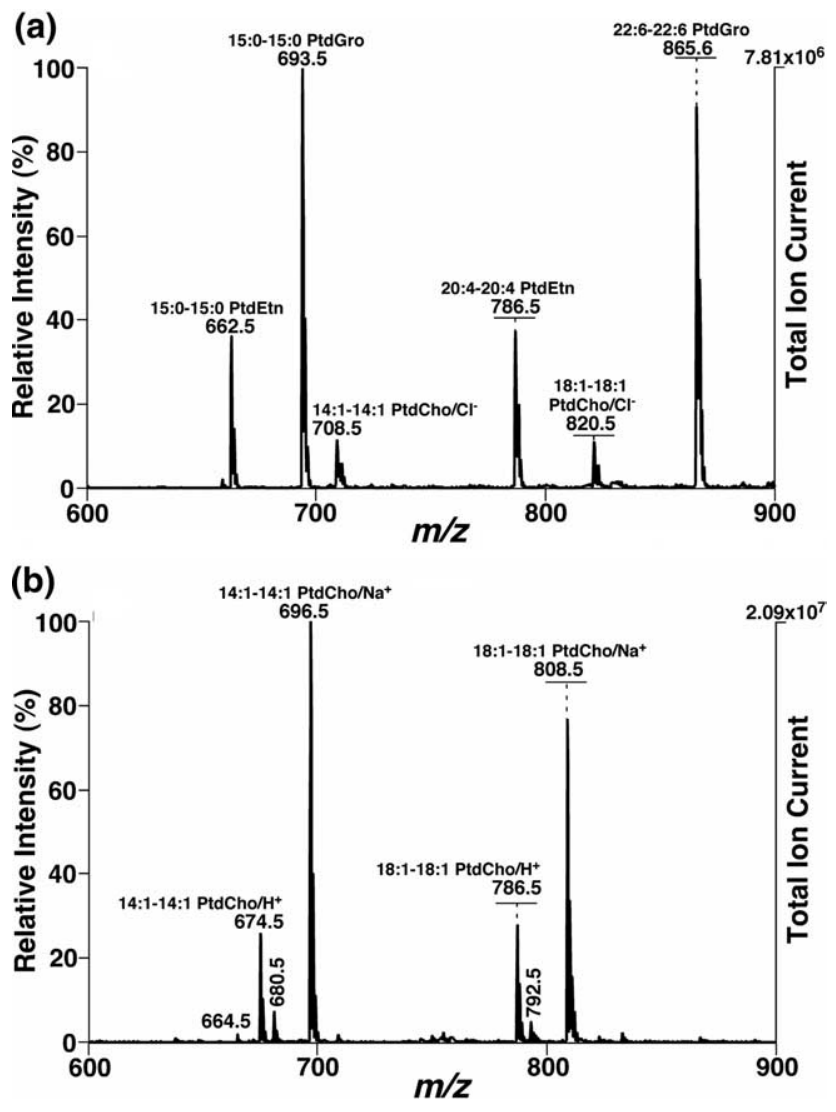

Figure 1. Representative ESI mass spectra of a phospholipid mixture in the negative- and positive-ion modes. The phospholipid mixture was comprised of $1 \mathrm{pmol} / \mu \mathrm{L}$ each of 15:0-15:0 and 22:6-22:6 PtdGro, $10 \mathrm{pmol} / \mu \mathrm{L}$ each of 14:1-14:1 and 18:1-18:1 PtdCho, and $15 \mathrm{pmol} / \mu \mathrm{L}$ each of 15:0-15:0 and 20:4-20:4 PtdEtn molecular species in $1: 1$ (vol/vol) $\mathrm{CHCl}_{3} / \mathrm{MeOH}$. The mixture was diluted from a stock mixture solution of total $5.2 \mathrm{nmol} / \mu \mathrm{L}$ (which was back extracted against $50 \mathrm{mM} \mathrm{LiCl}$ by a modified Bligh and Dyer procedure) before infusion. The infusion flow rate was $8 \mu \mathrm{L} / \mathrm{min}$. The negative-ion ESI mass spectrum (a) and positive-ion ESI mass spectrum (b) were acquired using a TSQ ESI mass spectrometer as described under the Materials and Methods section. All the indicated molecular species were identified by product ion ESI/MS analysis. The ion peaks at $\mathrm{m} / \mathrm{z} 664.5,680.5$, and 792.5 in (b) correspond to the protonated 15:0-15:0 PtdEtn as well as the lithiated 14:1-14:1 and 18:1-18:1 PtdCho molecular species, respectively. The horizontal bars indicate the ion peak intensities after de-isotoping of ${ }^{13} \mathrm{C}$ isotopomers and normalization of molecular species in each lipid class to the one containing less carbon numbers (i.e., lower molecular weight).

through the identification of the presence of abundant fatty acyl carboxylates and specific fragment ions of head groups of different lipid classes [23] confirmed that the two abundant ion peaks were deprotonated PtdGro species and the four low-abundance peaks were deprotonated PtdEtn species and chlorinated PtdCho species as indicated (Figure 1a). The two molecular species in each individual class were present in equal intensities (within experimental error as obtained from replicate determinations) after de-isotoping of ${ }^{13} \mathrm{C}$ isotopomers $[12,28,29]$. For comparison, the results were normalized to molecular species containing less carbon 
numbers by showing the calculated height by horizontal bars (Figure 1a). Thus, these results verify that instrumental response factors of individual molecular species in each examined class were independent of the acyl chain length and the degree of unsaturation of the molecular species at the low concentrations employed [12] under these experimental conditions. The peak intensity ratio of PtdGro to PtdEtn molecular species was $\sim 2$ to 1 . Because the amount of PtdGro in the examined mixture was 15 times less than that of PtdEtn, we demonstrated $\sim 30$-fold selectivity for the ionization of PtdGro over PtdEtn.

After switching to the positive ion mode during the identical infusion, the ESI mass spectrum displayed an entirely different profile of ions (Figure $1 b$ ) with the base peak of $\sim 2.1 \times 10^{7}$ ion current. Two very abundant ion peaks were present as well as some low-abundance ion peaks. The two abundant ion peaks were identified as the sodiated PtdCho molecular species by tandem mass spectrometric analyses. Other ion peaks corresponded to the protonated PtdCho molecular species at $m / z 674.5$ and 786.5 as well as the lithiated PtdCho molecular species at $m / z 680.5$ and 792.5 (Figure $1 b)$. The very low-abundance ion peak at $\mathrm{m} / \mathrm{z} 664.5$ was the protonated 15:0-15:0 PtdEtn (Figure 1b). Three conclusions can be drawn from the analysis of this spectrum (Figure 1b). First, the total intensities of protonated, lithiated, and sodiated ions of each equimolar PtdCho molecular species are essentially identical within experimental error after de-isotoping of ${ }^{13} \mathrm{C}$ isotopomers. Second, the PtdCho species containing shorter acyl chain length favor the affinity with sodium whereas those containing longer acyl chain length prefer protonation (see discussion below). Third, exquisitely selective ionization of PtdCho molecular species over PtdGro and PtdEtn molecular species was manifest in the positive-ion mode.

To determine the factors involved in intrasource separation and selective ionization, identical phospholipid mixtures were analyzed again by ESI/MS in both negative- and positive-ion modes under alkaline conditions by addition of a small amount of $\mathrm{LiOH}$ in methanol (30 pmol $/ \mu \mathrm{L}$, approximately equal to the amount of PtdEtn species in the solution) just before infusion. ESI mass spectra showed very different profiles (Figure 2) compared to their neutral infusate counterparts (Figure 1). The negative-ion ESI mass spectrum displayed two intense ion peaks corresponding to the deprotonated PtdEtn species of equal intensity (within experimental error) with an ion current of $\sim 2.0 \times 10^{7}$ and two other low-abundance ion peaks corresponding to the deprotonated PtdGro species. The ion peak intensity ratios of PtdEtn to PtdGro species were $\sim 15: 1$, which is the molar ratio of these species in the mixture. A very low-abundance ion peak at $\mathrm{m} / \mathrm{z} 708.5$ was also present, corresponding to the chlorinated PtdCho molecular species (Figure 2a), demonstrating the selective ioniza-
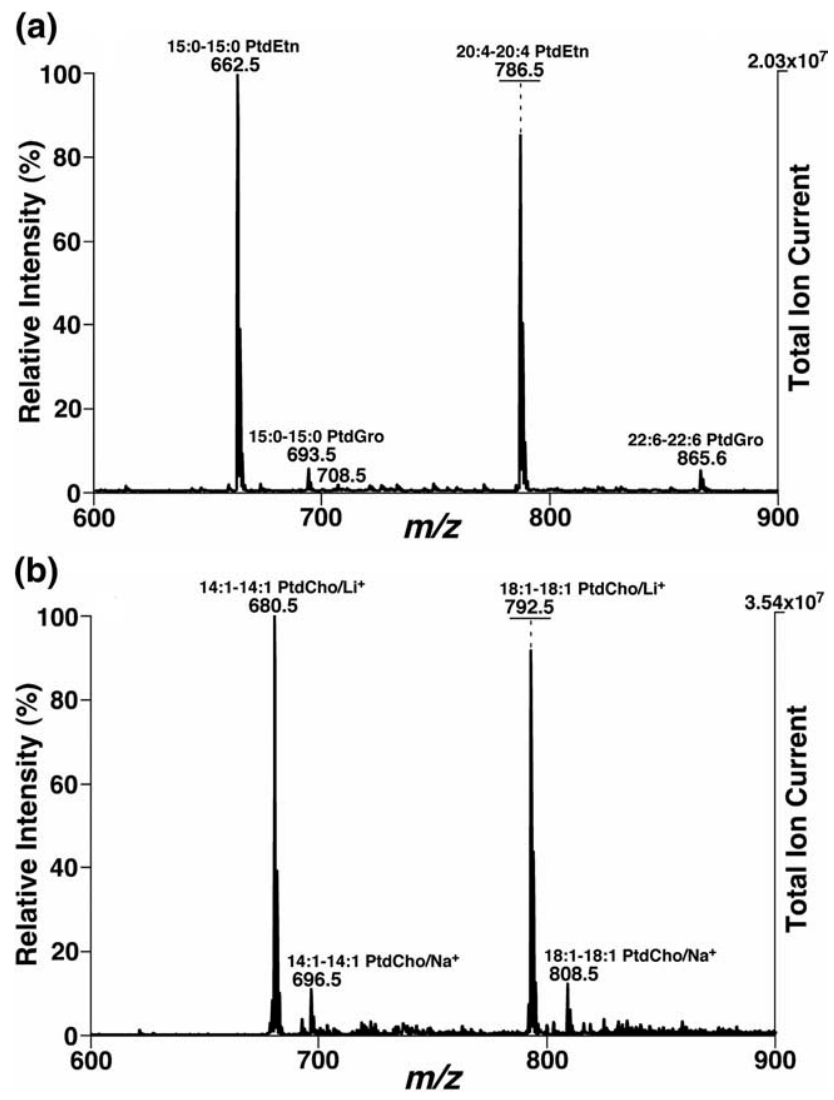

Figure 2. Representative ESI mass spectra of a phospholipid mixture in the negative- and positive-ion modes under alkaline conditions. All the experimental conditions were identical to those described in the legend of Figure 1 except that $30 \mathrm{pmol} / \mu \mathrm{L}$ of $\mathrm{LiOH}$ in methanol was added to the infusion solution immediately before infusion. All the indicated molecular species were confirmed by product ion ESI/MS analysis. The ion peak at $\mathrm{m} / \mathrm{z} 708.5$ in (a) corresponds to the chlorinated 14:1-14:1 PtdCho molecular species.

tion of the PC class from the other categories of lipids under the experimental conditions employed.

The positive-ion ESI mass spectrum showed two intense ion peaks which were identified as lithiated PtdCho molecular species with an ion current of $\sim 3.5 \times$ $10^{7}$ for the base peak. These ion peaks were also present at equal intensities (within experimental error) after de-isotoping of ${ }^{13} \mathrm{C}$ isotopomers, demonstrating again that the instrumental response factors of individual molecular species of the same class are not dependent on the acyl chain length and the degree of unsaturation of molecular species under the experimental conditions employed. Two low intensity ion peaks corresponding to the sodiated PtdCho molecular species were also present in the spectrum (Figure $2 \mathrm{~b}$ ). There were no ion peaks corresponding to protonated PtdCho molecular species under the experimental conditions. Collectively, these results demonstrate that PtdCho molecular species were selectively ionized by addition of a small amount of $\mathrm{LiOH}$ (approximately equal to the amount of PtdEtn in the infusion solution). 
The Effects of Infusion Flow Rates

and Phospholipid Concentrations on Selective Ionization

The selective ionization of PtdGro and PtdEtn species was well demonstrated in Figure 1a with $\sim 30$-fold resolution, resulting from the 15 -fold difference in mass content and over a 2-fold difference in ion peak intensity under the experimental conditions employed. These differences can be more directly and broadly examined by varying different factors such as the phospholipid concentration and the flow rate of an infusion solution when we define the selective ionization of two lipid classes as the ratio of the ionization efficiencies of these two classes, where the ionization efficiency of a class is defined as the normalization of the total ion current (or counts) to the total mass content of the class, that is

$$
\mathrm{IE}=\mathrm{TIC} / \sum_{\mathrm{i}=1}^{\mathrm{n}}\left[C_{\mathrm{i}}\right]
$$

where IE represents ionization efficiency of an examined lipid class; TIC denotes total ion current (or counts) of the class of interest; $\left[\mathrm{C}_{\mathrm{i}}\right]$ is the concentration of individual molecular species $i$ in the class;

$$
\sum_{\mathrm{i}=1}^{\mathrm{n}}\left[C_{\mathrm{i}}\right]
$$

stands for the total concentration of the class of interest, and $\mathrm{n}$ is the number of different molecular species in the class.

Figure 3a shows the ionization efficiency of PtdGro at the selected PtdGro concentrations of infused phospholipid solutions and flow rates of infusion. Although the ionization efficiencies of PtdGro increased as the flow rate increased at any given concentration of the examined phospholipid mixture, the increases in the ionization efficiencies were insignificant at the flow rates commonly employed in our laboratories for the analysis of biological samples ( $>4 \mu \mathrm{L} / \mathrm{min}$ ) (Figure $3 a$ ). The standard errors from the mean (SEM) of the calculated ionization efficiencies at any given concentration of the mixture were well within the experimental error typically present in biological experiments $(\leq 5 \%)$ (denoted as the broken line in Figure 3b). Interestingly, the effects of the flow rate on the ionization efficiencies of $\mathrm{PE}$ and PC classes at any given concentration in the infusion mixture employed were minimal (Figure $3 b$ ). These results indicate that the selective ionization of PtdGro from PtdEtn and PtdCho ((35 \pm 4$)$-fold and (120 \pm 12 )-fold, respectively), was constant within experimental error for the given instrument under a wide range of experimental conditions employed. The selective ionization of PtdGro from PtdCho and PtdEtn molecular species was also examined by varying the molar ratio of phospholipid constituents. The selective ionization of PtdGro from PtdCho and PtdEtn was essentially identical to those obtained from a mixture at (a)

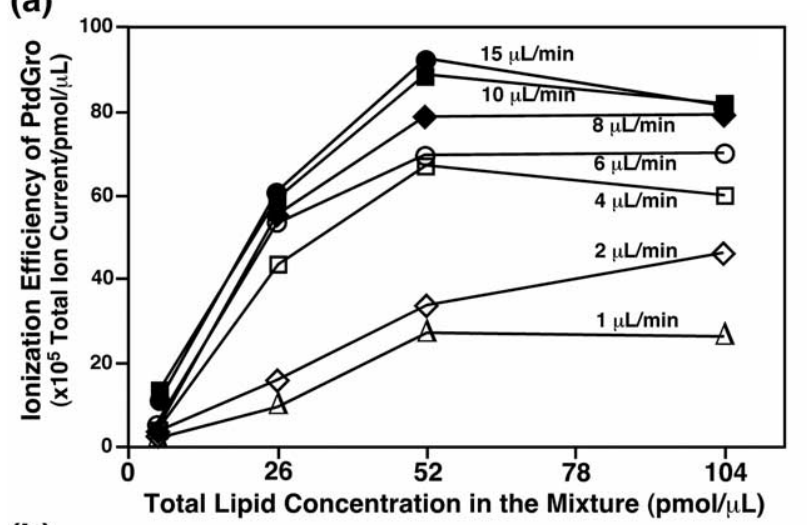

(b)

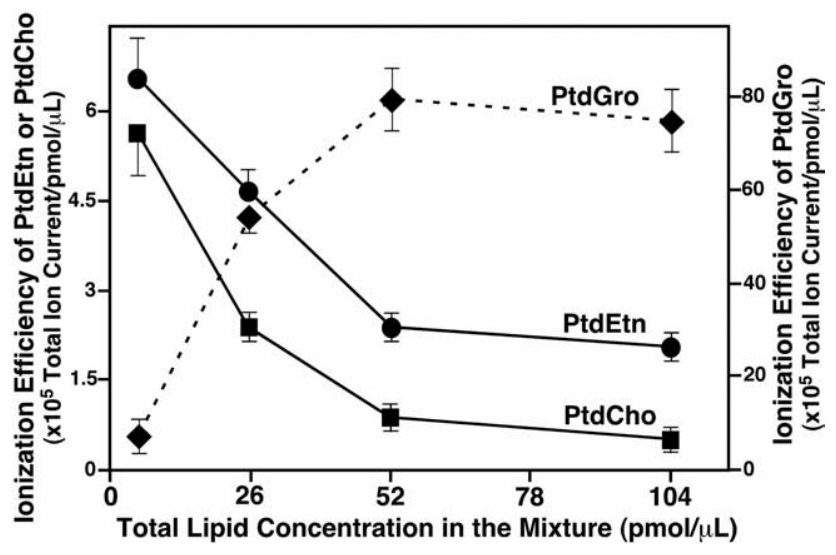

Figure 3. The effects of flow rate and lipid solution concentration on the normalized ion count density (i.e., ionization efficiency) of each examined phospholipid class in the negative-ion mode. The phospholipid mixture was comprised of 15:0-15:0 and 22:6-22:6 PtdGro, 14:1-14:1 and 18:1-18:1 PtdCho, and 15:0-15:0 and 20:420:4 PtdEtn molecular species in a molar ratio of 1:1:10:10:15:15 in 1:1 (vol/vol) $\mathrm{CHCl}_{3} / \mathrm{MeOH}$. Ion intensities in ion current of each phospholipid molecular species were determined from ESI/MS analyses of three independent injections using a TSQ ESI mass spectrometer. The ionization efficiencies of PtdGro [(a) and the broken line in (b)], PtdCho (b), and PtdEtn (b) were calculated from the normalization of the total ion current of a class to a unit concentration (i.e., pmol $/ \mu \mathrm{L}$ ) of each class at the indicated lipid concentration and flow rate. The ionization efficiency of PtdEtn or PtdCho represents the mean \pm SD of the ionization efficiencies of the class at the flow rates of $1,2,4,6,8,10$, and $15 \mu \mathrm{L} / \mathrm{min}$. The ionization efficiency of PtdGro in (b) represents the mean \pm SD of the ionization efficiencies in (a) at the flow rates of 4, 6, 8, 10, and $15 \mu \mathrm{L} / \mathrm{min}$. Some of the error bars are within the symbols.

the ratio of 1:10:15 of PtdGro/PtdCho/PtdEtn after calculation of the molar ratio as long as the mixture contained over $2 \mathrm{pmol} / \mu \mathrm{L}$ of PtdGro molecular species.

The effects of the lipid concentrations on the selective ionization of anionic lipid classes from PE and PC classes are intriguing. At the low concentration such as $0.1 \mathrm{pmol} / \mu \mathrm{L}$ of each PtdGro molecular species in the examined mixture, the contribution of each phospholipid class to the spray current (i.e., ionization efficiency) was essentially identical and independent of the infusion flow rates. The contributions of PtdGro, Pt$\mathrm{dEtn}$, and PtdCho were $(7 \pm 1) \times 10^{5},(6.6 \pm 0.4) \times 10^{5}$, 
and $(5.6 \pm 0.3) \times 10^{5}$ ion current $/ \mathrm{pmol} / \mu \mathrm{L}$, respectively, on the TSQ mass spectrometer (Figure 3b). This result indicates that at the low concentrations employed, the solution behaves as an "ideal" solution and each molecular species that exists in solution has an equal chance to be ionized to maintain the minimal faradic current. As the concentration of analytes in the infusion solution increased, the ionization efficiency of PtdGro exponentially increased whereas those of PtdEtn and PtdCho concordantly decreased, indicating an augmentation of the ionization selectivity of PtdGro molecular species accompanied by the reduction of the ionization efficiency of less-readily ionizable coexisting molecular species such as PtdEtn and PtdCho to achieve a maximal spray current.

This augmentation in ionization selectivity is consistent with a donor-acceptor model (e.g., conjugate acidconjugate base model) in which PtdGro molecular species can generate their conjugate base by losing a proton [or a small cation (e.g., $\mathrm{Li}^{+}, \mathrm{Na}^{+}$, etc.)] whereas both PtdCho and PtdEtn molecular species behave as a conjugate base relative to PtdGro to accept the proton (or small cation). Although PtdEtn and PtdCho species (as well as other small ions present in the media) in addition to anionic phospholipids are present in the initial sprayed droplets in the negative-ion mode under (very) weak acidic conditions, anionic lipids are present in greater abundance since anionic lipid molecular species form their conjugate bases more readily than either PtdCho or PtdEtn molecular species. This preferred selectivity of anionic species over PtdCho and PtdEtn species is also likely present during the iterative processing of desolvation and reformation of smaller droplets. This selectivity is likely achieved through a small cation transferring from a neutral anionic phospholipid molecular species to PtdCho or PtdEtn molecular species. This transfer leads to a charge redistribution preventing PtdEtn and PtdCho molecular species from entering the plate orifice (or other ion inlets). The augmentation of ionization selectivity of PtdGro over PtdCho and PtdEtn represents the cumulative effects resulting from this preferred selectivity during charged droplet formation as well as electric field dependent selectivity at each step of the droplet desolvation and reformation process.

To examine this hypothesis, we determined the ionization efficiency of individual PtdGro or PE class. We found that the ionization efficiency of PtdGro class alone was constant [i.e., $(7.9 \pm 0.6) \times 10^{5}$ ion current/ $\mathrm{pmol} / \mu \mathrm{L}]$ over a 1000 -fold concentration range examined $(0.01$ to $10 \mathrm{pmol} / \mu \mathrm{L})$. This ionization efficiency of PtdGro alone was comparable to that of PtdGro at its very low concentrations in the mixture and was $\sim 10$ times lower than that at its high concentration in the mixture examined (Figure 3b). The ionization efficiency of PtdEtn alone at its very low concentrations is comparable to that in the mixture. In contrast to PtdGro, as the concentration of PtdEtn increased, the ionization efficiency of PtdEtn becomes smaller. The ionization efficiency of PtdEtn averaged $(4.5 \pm 0.5) \times 10^{5}$ ion current $/ \mathrm{pmol} / \mu \mathrm{L}$ from a concentration range between 1 and $30 \mathrm{pmol} / \mu \mathrm{L}$ and this value was approximately two-fold higher than that obtained from the measurement of the phospholipid mixture (Figure 3b). Similarly, an average ionization efficiency of $(3.4 \pm 1.2) \times$ $10^{5}$ ion current $/ \mathrm{pmol} / \mu \mathrm{L}$ from PtdCho in this concentration range was also obtained. Again, this value was much higher that than obtained from the measurement of the phospholipid mixture (Figure $3 b$ ). These results suggest that the augmentation of PtdGro ionization in the phospholipid mixture is related to a dramatic reduction of PtdEtn and/or PtdCho ionization due to the favorable association of small cations with PtdEtn or PtdCho molecular species in comparison to PtdGro species in each step of the droplet processing.

\section{The Effects of the Number of Different Molecular Species in an Examined Phospholipid Class on Selective Ionization}

It was demonstrated in the last subsection that the ionization efficiencies of PtdCho, PtdEtn, and PtdGro are independent of lipid concentration and infusion flow rate under the examined experimental conditions. Therefore, the number of different molecular species in an examined phospholipid class should not affect the selective ionization of this class (in aggregate) from the other lipid classes of interest. Since ionization of each individual molecular species of a phospholipid class in the low concentration region is largely independent of the physical properties (e.g., chain length and degree of unsaturation) of individual molecular species, eq 1 can be written as

$$
\mathrm{IE} \approx \mathrm{IC}_{\mathrm{i}} /\left[\mathrm{C}_{\mathrm{i}}\right]
$$

where $\mathrm{IC}_{\mathrm{i}}$ is the ion current (or counts) of molecular species i. In other words, the ion peak intensity of an individual molecular species of the class proportionally depends on the concentration of the individual molecular species. From eq 2, clearly, at a certain total concentration of a class in a lipid mixture, the ion peak intensity of an individual molecular species in that class is inversely proportional to the number of different abundant individual molecular species comprising the class of interest in the infusion solution. Accordingly, comparisons with internal standards of that class can be directly used.

From this logical derivation, an interesting and useful phenomenon can be recognized. That is, alterations in the number of different abundant individual molecular species in independent classes can effect the apparent selective ionization (defined as the ionization efficiency ratio of the most abundant species in each lipid class of interest). Thus, if the number of different abundant individual molecular species in PE class is greater than that in PtdGro class when the molar ratio of PtdEtn versus PtdGro is constant, mass spectral 

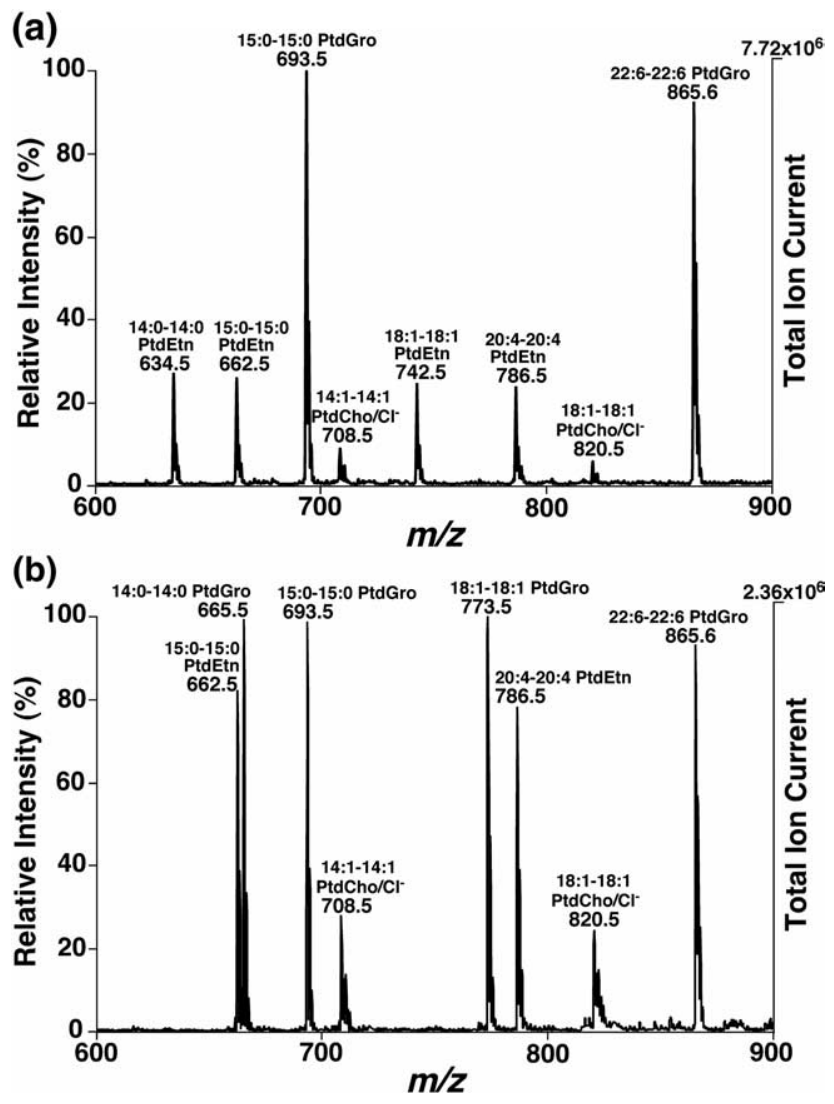

Figure 4. The effects of the number of different molecular species of a class on intrasource separation in the negative ion mode. The phospholipid mixture used was identical to that used in the Figure 1 except that two additional PtdEtn molecular species (a) or two additional PtdGro molecular species (b) were included. The mixture was diluted from a stock mixture solution which was back extracted against $50 \mathrm{mM} \mathrm{LiCl}$ by a modified Bligh and Dyer procedure before infusion. The infusion flow rate was $8 \mu \mathrm{L} / \mathrm{min}$. The negative-ion ESI mass spectrum was acquired using a TSQ ESI mass spectrometer. All the indicated molecular species were confirmed by product ion ESI/MS analysis.

analysis will show a greater apparent selective ionization than that manifested for the whole class [e.g., (35 \pm 4)-fold for PtdEtn versus PtdGro]. In contrast, if the number of different abundant individual molecular species in PtdEtn class is less than that present in PtdGro molecular species, mass spectral analysis will show a smaller apparent selective ionization than that manifested for the whole class.

This phenomenon can be demonstrated by inclusion of additional individual molecular species of a phospholipid class of interest into the original phospholipid mixture. For example, inclusion of two individual PE molecular species (14:0-14:0 and 18:1-18:1 PtdEtn) into the original phospholipid mixture resulted in the lower peak intensities of individual PtdEtn molecular species relative to the ion peak intensities from the analysis of the original phospholipid mixture containing only 2 PtdEtn molecular species (Figure 4a), compared to Figure 1a). This results in a calculated selective ionization of $\sim 60$-fold for any pair of PtdEtn and PtdGro molecular species. In contrast, inclusion of two different PtdGro molecular species (14:0-14:0 and 18:1-18:1 PtdGro) into the original phospholipid mixture yielded the lower peak intensities of each individual PtdGro molecular species relative to the ion peak intensities from the analysis of the original phospholipid mixture (Figure 4b, compared to Figure 1a). This result leads to an apparent selective ionization of $\sim 20$-fold for any pair of PtdEtn and PtdGro molecular species.

Practically, this relationship between the apparent selective ionization and the number of different individual molecular species in a class of interest is very useful for the analysis of biological samples based on intrasource separation and selective ionization because in general, the number of different abundant individual PE molecular species is always substantially larger than that of different abundant anionic phospholipid molecular species in biological samples [21, 22, 30, 31]. Therefore, an 100-fold apparent selective ionization of anionic phospholipid molecular species from PE molecular species was frequently observed by comparison of the difference between internal standards for the analyses of PE and anionic phospholipids as previously demonstrated [21].

\section{The Effects of Alkalization of the Infusion Solution on Intrasource Separation}

Lipid profiles acquired in both negative- and positiveion modes (Figure 1) were totally different (Figure 2) when the identical solution was analyzed after addition of a small amount of $\mathrm{LiOH}$. The next experiments were designed to identify the factors leading to differential ionization of lipid classes during intrasource separation after alkalization of the infusion solution. By stepwise addition of $\mathrm{LiOH}$, alterations in ionization efficiencies of phospholipid classes were examined by ESI/MS analysis of a phospholipid mixture comprised of two molecular species from each of PtdGro, PtdCho, and PtdEtn classes at a molar ratio of 1:10:15 and a total concentration of $26 \mathrm{pmol} / \mu \mathrm{L}$. ESI/MS analysis of the phospholipid mixture in the negative-ion mode demonstrated the decrease of the ion peaks corresponding to the PtdGro molecular species when the infusion solution was alkalized. This occurred because PtdEtn molecular species were rendered anionic after addition of LiOH and the ionization efficiency of anionic PtdEtn species was similar to that of anionic phospholipids [13, 21, 22]. In each of these and many other studies, the peak intensity ratio of PtdEtn and PtdGro (internal standards) was near the molar ratio added during sample preparation. Similar results were also obtained by addition of small amounts of other alkaline media including ammonium hydroxide. These results indicate that the presence of an alkaline component (even a small amount of weak alkaline media) can dramatically affect the intrasource separation of anionic phospholip- 
ids from PtdEtn molecular species attributable to formation of the conjugate base of PtdEtn.

Many studies have demonstrated that PtdEtn molecular species can readily be analyzed by negative-ion ESI/MS [23, 32-35] since these species become negatively charged after the facile loss of a proton from the head group of PtdEtn under alkaline conditions (i.e., conjugate acid). Thus, there are some similarities in electrical properties between ethanolamine glycerophospholipids and anionic phospholipids when alkali media conditions are employed. However, regardless of the similarity between these phospholipid classes, selective ionization can be achieved with an $\sim 35$-fold difference between the ion intensities of these two classes in the absence of alkali.

The analysis of the negative-ion ESI mass spectrum also showed the disappearance of ion peaks corresponding to chlorinated PtdCho molecular species as the concentration of $\mathrm{LiOH}$ increased in the infusion solution. This observation likely resulted from the combination of selective ionization of PtdEtn under the stated conditions and a reduced availability of chloride to PtdCho molecular species in the presence of hydroxide. Interestingly, no ion peaks corresponding to the hydroxyl adducted PtdCho molecular species were present in the spectrum, even at the highest concentration of $\mathrm{LiOH}$ examined, indicating that unlike chloride or acetate or other small anions, the affinity of hydroxide to PtdCho molecular species is very weak. These results also indicate that introduction of alkaline species in the infusion solution can enhance the intrasource separation of PtdEtn molecular species from their PtdCho counterparts in the negative-ion mode.

When the phospholipid mixture was analyzed in the positive-ion mode, the mass spectrum showed that the substantive ion peaks were comprised of the various adducts of PtdCho molecular species (Figure 1b), indicating the selective ionization of PtdCho molecular species from other classes of lipids. These mass spectra also showed that the disappearance of protonated ion peaks due to the neutralization of protons by $\mathrm{LiOH}$ and the appearance of lithiated ion peaks due to the increased availability of lithium ions in the infusion solutions after stepwise addition of $\mathrm{LiOH}$. The persistent presence of ion peaks in the spectrum corresponding to sodiated PC molecular species even at the relatively high concentration of $\mathrm{LiOH}$ suggests either the ready availability of sodium ions in the system or a high affinity of sodium ions with PtdCho molecular species or both.

The effects of infusion flow rates and solution concentrations on the intrasource separation and selective ionization of PtdCho species from PtdGro and PtdEtn species were examined by negative- and positive-ion ESI/MS analyses after addition of $\mathrm{LiOH}$. Variations in the infusion flow rates (from 1 to $15 \mu \mathrm{L} / \mathrm{min}$ ) and solution concentrations of PtdCho (from 0.2 to $40 \mathrm{pmol} /$ $\mu \mathrm{L})$ had no effect on these intrasource separations. Essentially identical results as shown in Figure 2 were obtained. That is, PtdCho molecular species as lithium adducts were specifically ionized in the positive-ion mode whereas ion peaks corresponding to PtdCho molecular species were markedly diminished in the negative-ion mode in the presence of a small amount of $\mathrm{LiOH}$ (approximately equal to the concentration of PtdEtn in the infusion solution).

\section{The Effects of Residual Ions in an Infusion Solution on Intrasource Separation and Selective Ionization}

Next, the effects of residual ions in an infusion solution from aqueous solutions on intrasource separation and selective ionization were examined using an identical phospholipid mixture as used in the last subsections at a total concentration of $26 \mathrm{pmol} / \mu \mathrm{L}$. The residual ions were the combinations of ions in the aqueous phase that remained during the back extraction of lipids in the chloroform layer. Four different aqueous media were employed during back extraction of the phospholipid mixture into chloroform: $50 \mathrm{mM} \mathrm{LiCl}, 50 \mathrm{mM}$ ammonium acetate, $50 \mathrm{mM} \mathrm{NH}_{4} \mathrm{Cl}$, and tenfold diluted PBS. It was found that there was no influence of the matrix ions on the intrasource separation and selective ionization that was demonstrated in Figures 1 and 2. However, there were some effects on the apparent phospholipid profiles when the phospholipid extracts were analyzed in both the positive- and the negative-ion modes without addition of $\mathrm{LiOH}$. For example, the negative-ion ESI mass spectrum of phospholipids extracted against ammonium acetate showed extra ion peaks corresponding to acetate adducts of PtdCho molecular species in addition to the chlorinated ion peaks. Figure 5 shows the positive-ion ESI mass spectra of these phospholipid extracts in which sodiated PtdCho peaks always dominated in comparison to protonated PtdCho peaks. The presence of abundant sodiated PtdCho peaks from all extracts even after extensive washing of the system suggests either the ready availability of sodium ions in the system itself or a high affinity of sodium ions for PtdCho molecular species or both. Another observation was that the presence of ion peaks corresponding to lithiated PC molecular species from phospholipids extracted against $\mathrm{LiCl}$ solutions was in low abundance, suggesting that the cations carried over during phospholipid extraction were in low concentration under the experimental conditions and that the proton and sodium ions were likely obtained from the sample inlet apparatus.

\section{Dependence of Intrasource Separation and Selective Ionization on the Ion Source}

The dependence of intrasource separation and selective ionization on the instrument employed was also examined using a different ion source to determine if the ion source design is a critical factor in the general applica- 
(a)

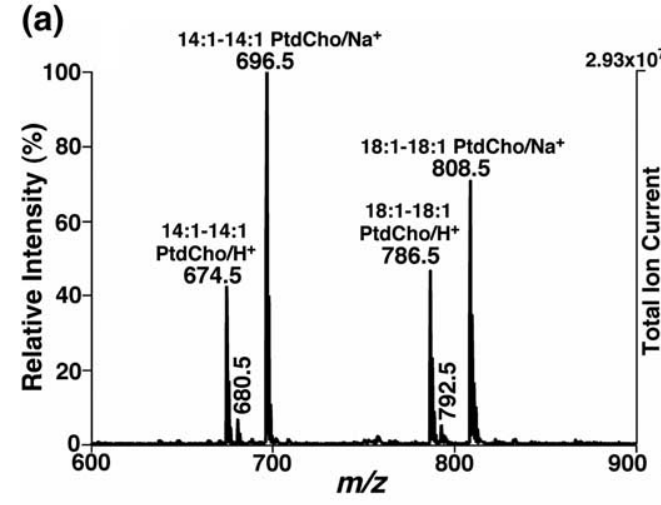

(c)

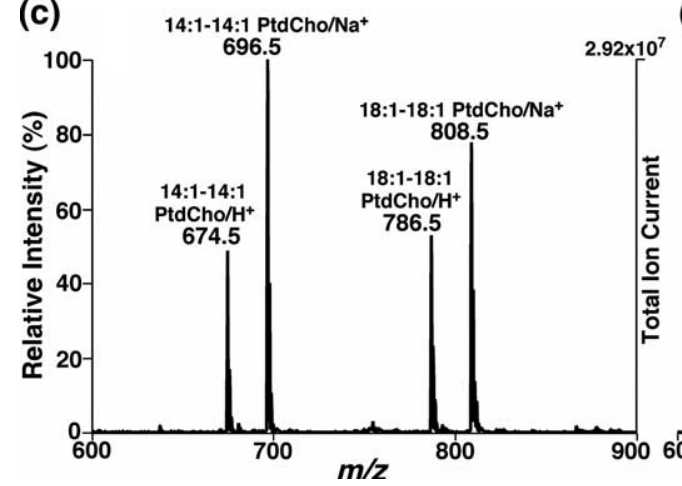

(b)

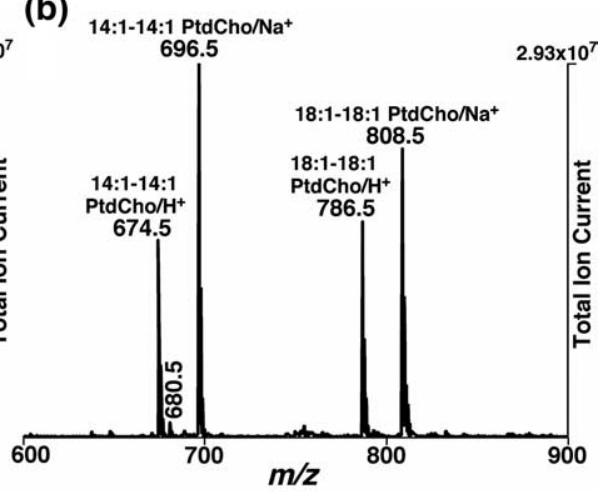

(d)

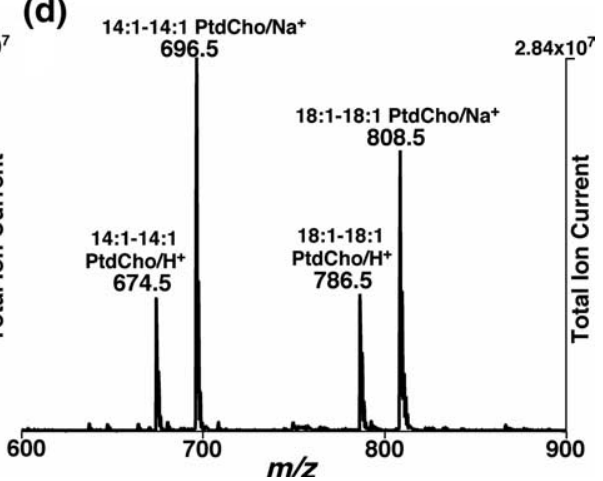

Figure 5. The effects of residual ions on intrasource separation in the positive ion mode. The lipid mixture was comprised of $0.5 \mathrm{pmol} / \mu \mathrm{L}$ each of 15:0-15:0 and 22:6-22:6 PtdGro, $5 \mathrm{pmol} / \mu \mathrm{L}$ each of 14:1-14:1 and 18:1-18:1 PtdCho, and 7.5 pmol/ $\mu$ L each of 15:0-15:0 and 20:4-20:4 PtdEtn in 1:1 (vol/vol) $\mathrm{CHCl}_{3} / \mathrm{MeOH}$. The mixture was diluted from a stock mixture solution of total $5.2 \mathrm{nmol} / \mu \mathrm{L}$ before infusion at a flow rate of $5 \mu \mathrm{L} / \mathrm{min}$. The stock mixture solution was back extracted against $50 \mathrm{mM} \mathrm{LiCl}$ (a), $50 \mathrm{mM}$ ammonium acetate (b), $50 \mathrm{mM} \mathrm{NH}_{4} \mathrm{Cl}$ (c), and tenfold diluted PBS (d) by a modified Bligh and Dyer procedure. Positive-ion ESI mass spectra were acquired using a TSQ ESI mass spectrometer as described in the Materials and Methods section. All the indicated molecular species were confirmed by product ion ESI/MS analysis. The ion peaks at $m / z 680.5$ and 792.5 in (b) correspond the lithiated 14:1-14:1 and 18:1-18:1 PtdCho molecular species, respectively.

bility of intrasource separation and selective ionization. ESI/MS analyses of a phospholipid mixture of PtdGro, PtdCho, and PtdEtn (two molecular species of each) at a constant ratio of 1:10:15 at the selected concentrations and infusion flow rates using a QqTOF mass spectrometer equipped with an ionspray source were performed. It was found that the intrasource separation and selective ionization of PtdEtn and PtdCho molecular species in the negative- and positive-ion modes, respectively, after addition of a small amount of $\mathrm{LiOH}$ was identical to that demonstrated by the ESI TSQ mass spectrometer used above. The selective ionization of anionic phospholipids from other categories of phospholipids (e.g., PtdCho and PtdEtn) in the negative-ion mode without addition of $\mathrm{LiOH}$ was also demonstrated by this instrument (Figure 6, compared to Figure 3b).

Similar to the results obtained from the TSQ mass spectrometer, the changes in ionization efficiency of PtdGro determined by the QqTOF mass spectrometer were very small in the low concentration regime of PtdGro in the mixture. At total PtdGro concentration above $2 \mathrm{pmol} / \mu \mathrm{L}$, the ionization efficiency of PtdGro increased slowly at the low flow rate of infusion, demonstrated a large change between 8 and $10 \mu \mathrm{L} / \mathrm{min}$ and significantly increased at a flow rate of $10 \mu \mathrm{L} / \mathrm{min}$ or higher. Again, the ionization efficiencies of PE and PC classes at any given concentration of the infused mixture were independent on the infused flow rates as found using the TSQ instrument (Figure 6b). Surprisingly, unlike results with the analyses by the TSQ instrument, the ionization efficiency of the PC class was not lower but slightly higher than that of PE (Figure 6b). Therefore, an 30-fold selection of PtdGro from both PtdEtn and PtdCho at a flow rate of $15 \mu \mathrm{L} / \mathrm{min}$ or higher and a PtdGro concentration over $1 \mathrm{pmol} / \mu \mathrm{L}$ can be obtained using the QqTOF mass spectrometer.

Many factors influencing the ionization conditions such as the solvent system employed, sheath gas flow rate, ionization temperature, etc. that might effect the overall ionization efficiencies of phospholipid classes and thus more or less affect the selective ionization. Since the ionization efficiencies of PtdGro, PtdCho, and PtdEtn are essentially independent of infusion flow rate and phospholipid concentration under most experimental conditions, alterations in most of these factors to certain degree do not significantly change the intra- 

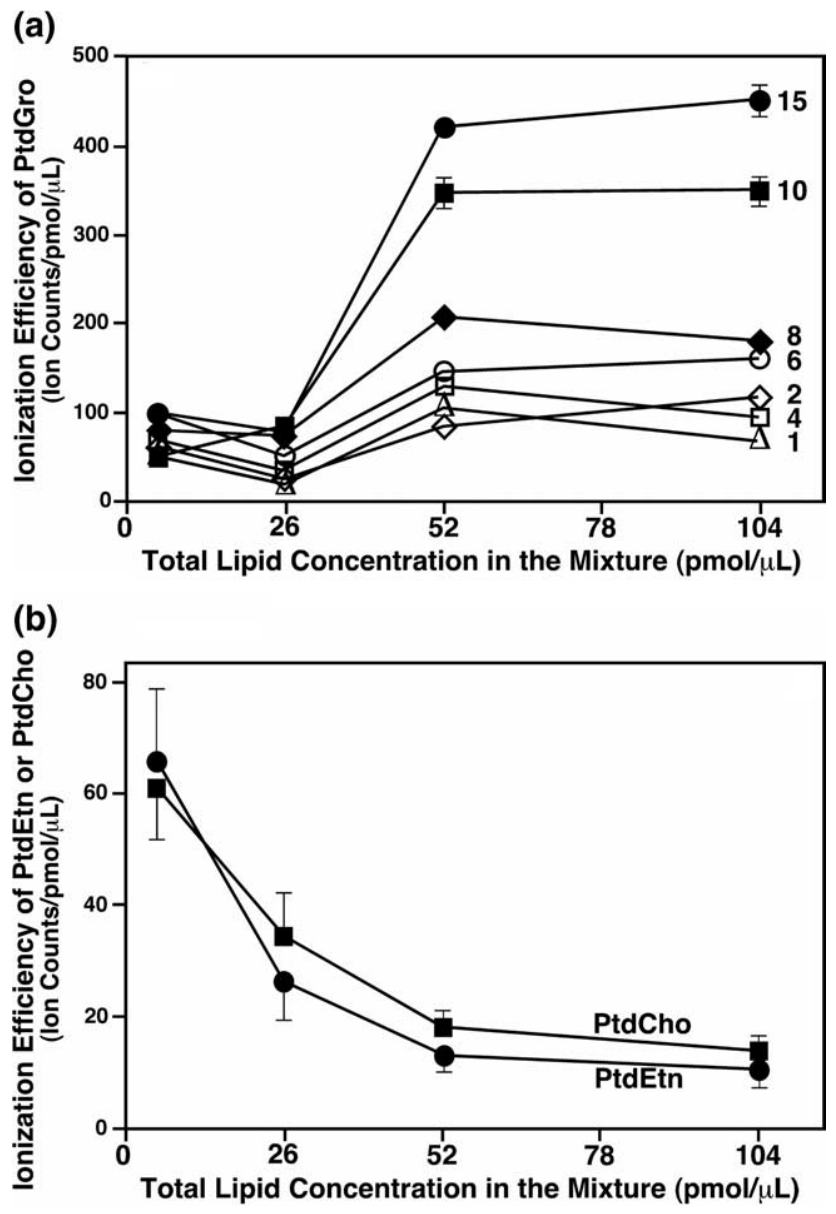

Figure 6. The effects of infusion flow rate and phospholipid concentration on ionization efficiencies of PtdCho, PtdEtn, and PtdGro in the negative-ion mode analyzed by a QqTOF mass spectrometer. All the experimental conditions employed were identical to those as described in the legend of Figure 3 except that ESI/MS analysis was performed using a QqTOF mass spectrometer as described in the Materials and Methods section. The numbers in (a) indicate the flow rates of infusion in $\mu \mathrm{L} / \mathrm{min}$. Some error bars are within the symbols.

source separation. This statement was verified by varying capillary temperature in a range of $\pm 50^{\circ} \mathrm{C}$, spray potential in a range of $\pm 500 \mathrm{v}$, and sheath gas pressure in a range of \pm 1 arbitrary unit on the TSQ instrument.

\section{Summary}

Through the manipulation of the physicochemical processes occurring in the ESI ion source during direct infusion, lipid classes can be selectively ionized in the ion source based on the electrical properties of each lipid class. The class of PC molecular species can be selectively ionized from many other classes of lipids in the positive-ion mode after addition of $\mathrm{LiOH}$. The class of PE molecular species can be selectively analyzed in the negative-ion mode after addition of $\mathrm{LiOH}$, in which the mass content differences between anionic phospholipids and PE molecular species in most of biological samples virtually make the separation complete (>95\%) between these classes. This is because the ionization sensitivities of anionic phospholipids and deprotonated PE molecular species are essentially identical [13], and the mass content of anionic phospholipids is not abundant in biological samples. This study emphasized the selective ionization of anionic phospholipids from PtdEtn and PtdCho molecular species. It was demonstrated that through intrasource separation and selective ionization, PtdGro (a representative of anionic phospholipids including phosphatidylserine and phosphatidylinositol) can be resolved from PtdEtn and PtdCho molecular species with selectivity factors of $\sim 35$ - and 120-fold, respectively, on the TSQ mass spectrometer, whereas $\sim 30$-fold resolution for both on the QqTOF mass spectrometer is obtained. The nature of this selective ionization results from the selective reduction of ionization of less ionizable molecular species. Practically, the apparent selective ionization is much larger than those obtained in these model systems due to the larger number of prominent individual molecular species in PE in comparison to anionic phospholipids in biological samples. Collectively, the selective ionization of anionic phospholipid molecular species from other lipid classes in the negative-ion mode, in addition to the selective ionization of PC and PE molecular species after addition of a small amount of weak base, greatly facilitates global analyses of cellular lipidomes without the obligatory need for chromatographic separation.

\section{Acknowledgments}

This work was supported by P01HL57278 and the Neurosciences Education and Research Foundation.

\section{References}

1. Fenn, J. B.; Mann, M.; Meng, C. K.; Wong, S. F.; Whitehouse, C. M. Electrospray Ionization for Mass Spectrometry of Large Biomolecules. Science 1989, 246, 64-71.

2. Fenn, J. B. Electrospray Wings for Molecular Elephants (Nobel Lecture). Angew. Chem. Int. Ed. Engl. 42387120033894

3. Fenn, J. B.; Mann, M.; Meng, C. K.; Wong, S. F.; Whitehouse, C. M. Electrospray Ionization-Principles and Practice. Mass Spectrom. Rev. 1990, 9, 37-70.

4. Smith, R. D.; Loo, J. A.; Edmonds, C. G.; Barinaga, C. J.; Udseth, H. R. New Developments in Biochemical Mass Spectrometry: Electrospray Ionization. Anal. Chem. 1990, 62, 882-899.

5. Kebarle, P.; Tang, L. From Ions in Solution to Ions in the Gas PhaseThe Mechanism of Electrospray Mass Spectrometry. Anal. Chem. 1993, 65, 972A-986A.

6. De la Mora, J. F.; Van Berkel, G. J.; Enke, C. G.; Col, R. B.; MartinezSanchez, M.; Fenn, J. B. Electrochemical Processes in Electrospray Ionization Mass Spectrometry. J. Mass Spectrom. 2000, 35, 939-952.

7. Pozniak, B. P.; Cole, R. B. Negative Ion Mode Evolution of Potential Buildup and Mapping of Potential Gradients within the Electrospray Emitter. J. Am. Soc. Mass Spectrom. 2004, 15, 1737-1747.

8. Rohner, T. C.; Lion, N.; Girault, H. H. Electrochemical and Theoretical Aspects of Electrospray Ionization. Phys. Chem., Chem. Phys. 2004, 6, 3056-3068.

9. Tang, L.; Kebarle, P. Effect of the Conductivity of the Electrosprayed Solution on the Electrospray Current. Factors Determining Analyte Sensitivity in Electrospray Mass Spectrometry. Anal. Chem. 1991, 63, 2709-2715.

10. Ikonomou, M. G.; Blades, A. T.; Kebarle, P. Electrospray-Ion Spray: A Comparison of Mechanisms and Performance. Anal. Chem. 1991, 63, 1989-1998.

11. Gaskell, S. J. Electrospray: Principles and Practice. J. Mass Spectrom. 1997, 32, 677-688.

12. Han, X.; Gross, R. W. Shotgun Lipidomics: Electrospray Ionization Mass Spectrometric Analysis and Quantitation of the Cellular Lipidomes 
Directly from Crude Extracts of Biological Samples. Mass Spectrom. Rev. 2005, 24, 367-412.

13. Han, X.; Gross, R. W. Electrospray Ionization Mass Spectroscopic Analysis of Human Erythrocyte Plasma Membrane Phospholipids. Proc. Natl. Acad. Sci. U.S.A. 1994, 91, 10635-10639.

14. Han, X.; Gubitosi-Klug, R. A.; Collins, B. J.; Gross, R. W. Alterations in Individual Molecular Species of Human Platelet Phospholipids During Thrombin Stimulation: Electrospray Ionization Mass SpectrometryFacilitated Identification of the Boundary Conditions for the Magnitude and Selectivity of Thrombin-Induced Platelet Phospholipid Hydrolysis. Biochemistry 1996, 35, 5822-5832.

15. Gross, R. W.; Sobel, B. E. Isocratic High-Performance Liquid Chromatography Separation of Phosphoglycerides and Lysophosphoglycerides. J. Chromatogr. 1980, 197, 79-85.

16. Han, X.; Cheng, H. Characterization and Direct Quantitation of Cerebroside Molecular Species from Lipid Extracts by Shotgun Lipidomics. J. Lipid Res. 2005, 46, 163-175.

17. Han, X.; Yang, K.; Cheng, H.; Fikes, K. N.; Gross, R. W. Shotgun Lipidomics of Phosphoethanolamine-Containing Lipids in Biological Samples After One-Step in Situ Derivatization. J. Lipid Res. 2005, 46, $1548-1560$.

18. Han, X.; Gross, R. W. Shotgun Lipidomics: Multi-Dimensional Mass Spectrometric Analysis of Cellular Lipidomes. Exp. Rev. Proteom. 2005, 2, 253-264.

19. Gross, R. W. High Plasmalogen and Arachidonic Acid Content of Canine Myocardial Sarcolemma: A Fast Atom Bombardment Mass Spectroscopic and Gas Chromatography-Mass Spectroscopic Characterization. Biochemistry 1984, 23, 158-165.

20. Bligh, E. G.; Dyer, W. J. A Rapid Method of Total Lipid Extraction and Purification. Can. J. Biochem. Physiol. 1959, 37, 911-917.

21. Han, X.; Yang, J.; Cheng, H.; Ye, H.; Gross, R. W. Towards Fingerprinting Cellular Lipidomes Directly from Biological Samples by TwoDimensional Electrospray Ionization Mass Spectrometry. Anal. Biochem. 2004, 330, 317-331.

22. Han, X. Cheng, H. Mancuso, D. J. Gross, R. W. Caloric Restriction Results in Phospholipid Depletion, Membrane Remodeling, and Triacylglycerol Accumulation in Murine Myocardium. Biochemistry 2004, 43, $15584-15594$

23. Han, X.; Gross, R. W. Structural Determination of Picomole Amounts of Phospholipids Via Electrospray Ionization Tandem Mass Spectrometry. J. Am. Soc. Mass Spectrom. 1995, 6, 1202-1210.

24. Cullis, P. R.; Fenske, D. B.; Hope, M. J. Physical Properties and Functional Roles of Lipids in Membranes. In Biochemistry of Lipids,
Lipoproteins, and Membranes; Vance, D. E.; Vance, J., Eds.; Elsevier: Amsterdam, 1996, pp 1-33.

25. Koivusalo, M.; Haimi, P.; Heikinheimo, L.; Kostiainen, R.; Somerharju, P. Quantitative Determination of Phospholipid Compositions by ESIMS: Effects of Acyl Chain Length, Unsaturation, and Lipid Concentration on Instrument Response. J. Lipid Res. 2001, 42, 663-672.

26. Hermansson, M.; Uphoff, A.; Kakela, R.; Somerharju, P. Automated Quantitative Analysis of Complex Lipidomes by Liquid Chromatography/Mass Spectrometry. Anal. Chem. 2005, 77, 2166-2175.

27. DeLong, C. J.; Baker, P. R. S.; Samuel, M.; Cui, Z.; Thomas, M. J. Molecular Species Composition of Rat Liver Phospholipids by ESI-MS/ MS: The Effect of Chromatography. J. Lipid Res. 2001, 42, 1959-1968.

28. Han, X.; Gross, R. W. Quantitative Analysis and Molecular Species Fingerprinting of Triacylglyceride Molecular Species Directly from Lipid Extracts of Biological Samples by Electrospray Ionization Tandem Mass Spectrometry. Anal. Biochem. 2001, 295, 88-100.

29. Han, X. Characterization and Direct Quantitation of Ceramide Molecular Species from Lipid Extracts of Biological Samples by Electrospray Ionization Tandem Mass Spectrometry. Anal. Biochem. 2002, 302, 199212.

30. Cheng, H.; Xu, J.; McKeel, D. W., Jr.; Han, X. Specificity and Potential Mechanism of Sulfatide Deficiency in Alzheimer's Disease: An Electrospray Ionization Mass Spectrometric Study. Cell. Mol. Biol. 2003, 49, 809-818.

31. Han, X.; Cheng, H.; Fryer, J. D.; Fagan, A. M.; Holtzman, D. M. Novel Role for Apolipoprotein E in the Central Nervous System: Modulation of Sulfatide Content. J. Biol. Chem. 2003, 278, 8043-8051.

32. Kerwin, J. L.; Tuininga, A. R.; Ericsson, L. H. Identification of Molecular Species of Glycerophospholipids and Sphingomyelin Using Electrospray Mass Spectrometry. J. Lipid Res. 1994, 35, 1102-1114.

33. Kim, H. Y.; Wang, T. C.; Ma, Y. C. Liquid Chromatography/Mass Spectrometry of Phospholipids Using Electrospray Ionization. Anal. Chem. 1994, 66, 3977-3982.

34. Ramanadham, S.; Hsu, F. F.; Bohrer, A.; Nowatzke, W.; Ma, Z.; Turk, J. Electrospray Ionization Mass Spectrometric Analyses of Phospholipids from Rat and Human Pancreatic Islets and Subcellular Membranes: Comparison to Other Tissues and Implications for Membrane Fusion in Insulin Exocytosis. Biochemistry 1998, 37, 4553-4567.

35. Taguchi, R.; Hayakawa, J.; Takeuchi, Y.; Ishida, M. Two-Dimensional Analysis of Phospholipids by Capillary Liquid Chromatography/Electrospray Ionization Mass Spectrometry. J. Mass Spectrom. 2000, 35, 953-966. 